\title{
A new method for position control of a 2-DOF robot arm using neuro-fuzzy controller
}

\author{
JafarTavoosi $^{1^{*}}$, Afshar Shamsi Jokandan ${ }^{2}$ and Muhammad Amin Daneshwar ${ }^{3}$ \\ ${ }^{1}$ Department of Engineering, Ilam Branch, Islamic Azad University, Ilam, Iran \\ ${ }^{2}$ Faculty of Electrical and Computer Engineering, University of Tabriz, Tabriz, Iran \\ ${ }^{*}$ Department of Electrical Engineering, Mahabad Branch, Islamic Azad University, Mahabad, Iran
}

Jtavoosii65@yahoo.com

\begin{abstract}
Robot manipulators have become increasingly important in the field of flexible automation. So modeling and control of robots in automation will be very important. But Robots, as complex systems, must detect and isolate faults with high probabilities while doing their tasks with humans or other robots with high precision and they should tolerate the fault with the controller.This paper introduces a Neuro-Fuzzy Controller (NFC) for position control of robot arm. A five layer neural network is used to adjust input and output parameters of membership function in a fuzzy logic controller. The hybrid learning algorithm is used for training this network. In this algorithm, the least square estimation method is applied for the tuning of linear output membership function parameters and the error backpropagation method is used to tune the nonlinear input membership function parameters. The simulation results show that NFC is better and more robust than the PID controller for robot trajectory control.
\end{abstract}

Keywords:Robot arm, Hybrid Learning,Neuro-Fuzzy Controller(NFC)

Introduction

The wider application of automatic control has developedrapidly in recent years. The reason for this is thecomplexity of modem plant and the constraints imposed bythe increasing demand for higher quality products. Hence, the design of a controller which possesses learning capability becomes highly desirable. Robots are highly reliable, dependable and technologically advanced factory equipment. All commercial industrialrobots have two physically separate basic elements themanipulator arm and the controller. In addition toapplications like serial product lines, robots are assigned tomissions like waste treatment in nuclear reactors, data collection in space and underwater tasks which can be very risky for humans. In very close years, Robots used to application with very precision such as surgical. Therefore, robots must controlled very precision, for this goal, we must use modern controller such as intelligent controller.

Ankarali et al. (2010) used NFC for 3-DOF Scara Robot; three adaptive networks based fuzzy logic controllers were used in control strategy as NFCs but third controller for wrist ofrobot is ineffective to track desired circular tool trajectory. These controllers are designed by training and checking datasets obtained from PID control of scara robot.

Nassim Nikpay et al., (2010) used WRBF network and neuro-fuzzy network to control the hub position of Flexible Link Robot Arm. In this article, they have comparisons Neuro- Fuzzy controller, RBF controller, Fuzzy controller and LQR controller to control of hub angle and they have shown that, achieving desired performance with adaptive intelligent scheme and since fuzzy controller consumes less energy in comparison with LQR controller.Jo-Anne Tinget al. (2011) have shown complex robots such as humanoids, model-based control is highly beneficial for accurate tracking while keeping negative feedback gains low for compliance. They demonstrated the efficiency of their algorithm by applying it to a synthetic dataset, a 7 DOF robotic vision head and a 10 DOF robotic anthropomorphic arm.

Zafer Bingul \& Oguzhan Karahan (2011) introduced the PSO based tuning method for FLC and PID controller to control the given robot trajectory. The all parameters concerning the fuzzy controller and the PID controller were determined using PSO algorithm. Kuo-Ho Su et al. (2010) used Neuro- Fuzzy controller for a two autonomously driven wheeled robot and they showed Neuro- Fuzzy controller is successfully applied to control the driving motor and to balance the two-wheeled robot. Ouardalbrahim \& Wisama Khalil (2010) presented recursive solutions for obtaining the inverse and direct dynamic models of hybrid robots. The hybrid structure is made up of $\mathrm{n}$ parallel modules, which are serially connected to a fixed base. Srinivasan Alavandar \& Nigam (2008) used ANFIS to Inverse Kinematics Solution of 3DOF Planar Robot. In this paper, they illustrated the ANFIS is able to identification and control of 2-DOF and 3-DOF robot manipulator and trained ANFIS can be utilized toprovide fast and acceptable solutions of the inverse kinematics of robots.

Deepak Batra et al. (2009) used kinematics routine for the axis control of the robot using brushless DC motor drive. This study indicates in order to keep the robot around its operating point, but also for safety reasons, the data was collected using an experimental feedback control arrangement, which subsequently allows offline computations of the reference signals for the jointcontrollers.Jun Wuet al. (2010) give an overview of the existing work on dynamic parameter identification of serial and parallel robots. In this paper the modal of a robot is identified for model-based control and shows that parameter identification for model-based control of a robot arm is very important because in real robot, the
Research article

Clndian Society for Education and Environment (iSee)
"Control of robot arm"

http://www.indjst.org
Jafar Tavoosi et al. Indian J.Sci.Technol. 
dynamic of a robot is time-varying and parameter identification can be useful.

In this paper, we use Takagi-Sugeno Type NeuroFuzzy Network with hybrid learning algorithm for identification of robot then trained Neuro - Fuzzy Network is used for Neuro- Fuzzy controller for position control of 2-DOF robot arm.

\section{Dynamic model of the 2-DOF robot arm}

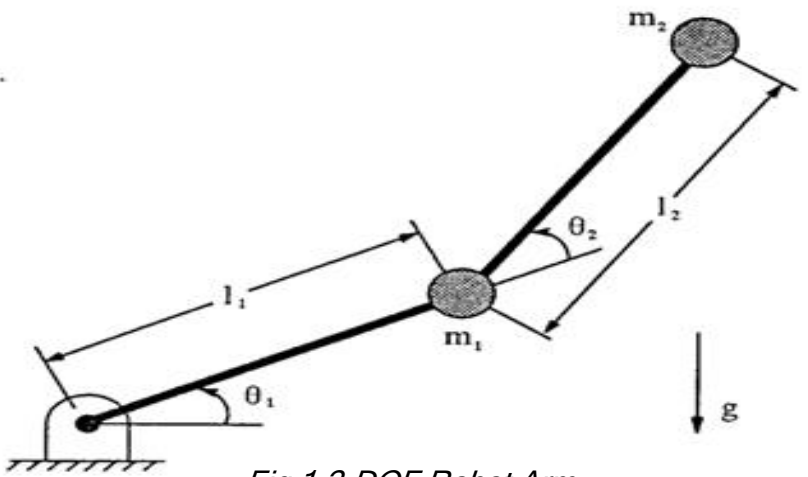

Fig 1.2-DOF Robot Arm

The dynamical analysis of the robot investigates a relation between the joint torques/forces applied by the actuators and the position,velocity and acceleration of the robot arm with respect to the time. Robot manipulators have complex non-linear dynamics that might make accurate and robust control difficult. Therefore, they are good examples to test performance of the controllers. The 2-DOF robotshown in Fig. 1 was selected as an example problem. The dynamicequations of the serial robot are usually represented by the following coupled non-linear differential equations:

$T=M(\Theta) \ddot{\Theta}+V(\Theta, \dot{\Theta})+G(\Theta)+F(\dot{\Theta})$

Where $T$ is Torque and $\Theta$ is position vector $\theta=$ $\left[\theta_{1}, \theta_{2}, \cdots, \theta_{n}\right]^{T}$, and $M(\Theta)$ is the $n \times n$ inertia matrix, $\mathrm{V}(\Theta, \dot{\Theta})$ is the $n \times 1$ coriolis/centripetalmatrix and $G(\Theta)$ is the $n \times 1$ gravitational toques and $F(\dot{\Theta})$ is the $n \times 1$ vector of dynamic and static friction forces

State space equation of robot arm with $x_{1}=\theta_{1}, x_{2}=\theta_{2}$, $x_{3}=\dot{\theta}_{1}, x_{4}=\dot{\theta}_{2}$ can be written as follows

$\dot{x}_{1}=x_{3}$

$\dot{x}_{2}=x_{4}$

$\dot{x}_{3}=\frac{1}{\Delta}\left[\left(l_{2}^{2} m_{2}\right)\left(\tau_{1}+2 \alpha s_{2} x_{3} x_{4}+\alpha x_{4}^{2}-\beta c_{1}-\gamma c_{12}\right.\right.$

$$
\left.-r_{1} x_{3}\right)
$$

$\left.-\left(l_{2}^{2} m_{2}+l_{1} l_{2} m_{2} c_{2}\right)\left(\tau_{2}-\alpha s_{2} x_{3}^{2}-\gamma c_{12}-r_{2} x_{4}\right)\right]$

$\dot{x}_{4}=\frac{1}{\Delta}\left[-\left(l_{2}^{2} m_{2}+l_{1} l_{2} m_{2} c_{2}\right)\left(\tau_{1}+2 \alpha s_{2} x_{3} x_{4}+\alpha x_{4}^{2}-\beta c_{1}\right.\right.$

$$
\left.-\gamma c_{12}-r_{1} x_{3}\right)
$$

$+\left(l_{1}^{2}\left(m_{1}+m_{2}\right)+l_{2}^{2} m_{2}+l_{1} l_{2} m_{2} c_{2}\right)\left(\tau_{2}-\alpha s_{2} x_{3}^{2}-\gamma c_{12}\right.$

$$
\left.\left.-r_{2} x_{4}\right)\right]
$$

Where, $c_{1}=\cos \left(\theta_{1}\right)$ and $c_{12}=\cos \left(\theta_{1}+\theta_{2}\right) . m_{1}, m_{2}$ are masses, $l_{1}, l_{2}$ are lengths and $r_{1}, r_{2}$ are Friction
Vol. 5 No.3 (Mar 2012)

ISSN: 0974- 6846

coefficients of joints, and $\Delta=l_{1}^{2} l_{2}^{2}\left[\left(m_{1}+m_{2}\right) m_{2}-m_{2}^{2} c_{2}^{2}\right]$, $\alpha=l_{1} l_{2} m_{2}, \beta=l_{1}\left(m_{1}+m_{2}\right) g, \gamma=l_{2} m_{2} g$

Fuzzy Neural Network

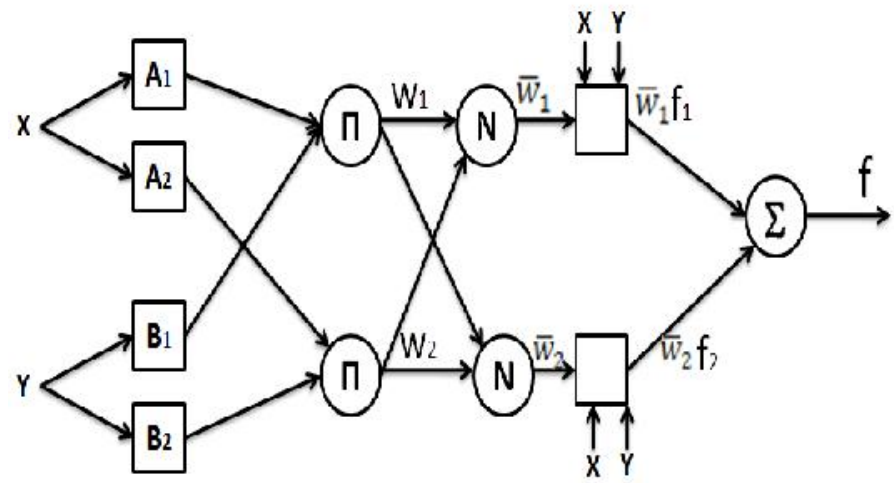

Fig.2 Structure of T-S Fuzzy Neural Network

In this study, adaptive network based fuzzy logic controller is applied for position control of robot arm. Established adaptive network based fuzzy inference system (ANFIS) uses a hybrid learning algorithm to identify parameters of Sugeno-type fuzzy inference system. It applies a combination of the least squares method and the back propagation gradientdescent method for training fuzzy inference system (FIS) membership function parameters to emulate a given training data set. The control algorithm proposed to be used in control of the 2-DOF Robot Arm is shown in Fig.1.

Before adaptation to the system, the fuzzy logic controller rule-base is optimized offline by using artificial neural network (ANN). These controllers are designed by training and checking data sets that are obtained from PID control of the system. First of all hierarchical PID controllers applied to the 2-DOF Robot Arm and then their inputs and outputs data are obtained to set up the adaptive network based fuzzy inference system (ANFIS). After training the network, fuzzy inference system structure is established. The network base is trained offline. Number of membership functions and type of membership functions are selected. Fig.2 illustrates Takagi-sugeno type of Fuzzy Neural Network. In this

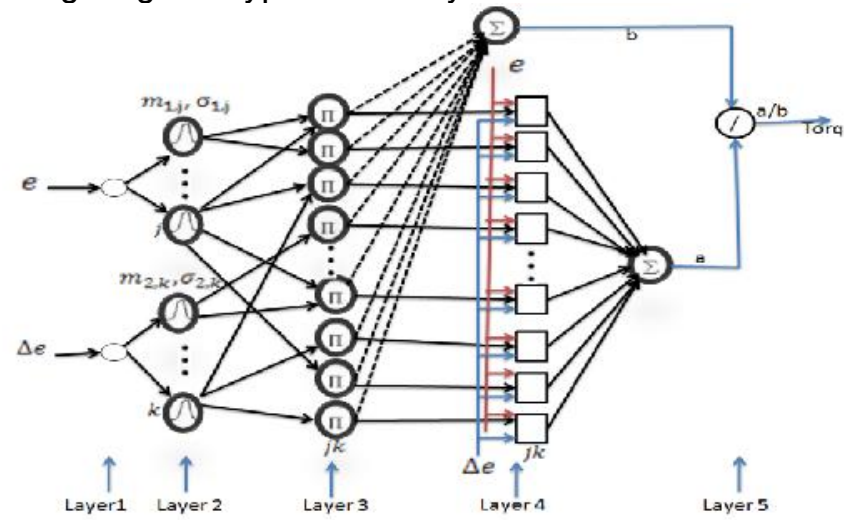

Fig 3. Structure of used Fuzzy Neural Network
Research article

COIndian Society for Education and Environment (iSee)
"Control of robot arm" http://www.indjst.org
Jafar Tavoosi et al. Indian J.Sci.Technol. 
model, output is linear equation of inputs. In Fig.2 we have two rule:

if $\mathrm{x}$ is $A_{1}$ and $\mathrm{y}$ is $B_{1}$ then $f_{1}=p_{1} x+q_{1} y+r_{1}$

if $\mathrm{x}$ is $A_{2}$ and $\mathrm{y}$ is $B_{2}$ then $f_{2}=p_{2} x+q_{2} y+r_{2}$

Neuro - fuzzy controller for 2-DOF robot arm

In this paper we have used the structure of Takagisugeno type of Fuzzy Neural Network in Fig.3.

In Fig.3, e and $\Delta e$ are inputs.In layer 1, each node performs a fuzzy set and the Gaussian function is adopted as membership function

$$
\begin{aligned}
& O_{1, i}^{1}=\mu_{A_{i}}(e)=\exp \left[-\left(\frac{e-m_{1, i}}{\delta_{1, i}}\right)^{2}\right] \\
& i=1,2, \ldots, j \\
& O_{2, l}^{1}=\mu_{B_{l}}(\Delta e)=\exp \left[\begin{array}{c}
-\left(\frac{\Delta e-m_{2, l}}{\delta_{2, l}}\right)^{2} \\
l=1,2, \cdots, k
\end{array}\right]
\end{aligned}
$$

The output of layer 2 as follows:

$$
O_{i l}^{2}=w_{i l}=\mu_{A_{i}} * \mu_{B_{l}}
$$

The output of layer 3 as follows:

$O_{i l}^{3}=w_{i l} f_{i l}=w_{i l}\left(p_{i l} e+q_{i l} \Delta e+r_{i l}\right)$

where $p_{i l}, q_{i l}$ and $r_{i l}$ are linear output parameters, that are determined by least square method.

In layer 4 ,

$a=\sum_{i} \sum_{l} w_{i l} f_{i l}$

$b=\sum_{i}^{i} \sum_{l}^{l} w_{i l}(8)$

$O_{0}^{4}=$ Torque

$=\frac{a}{b}$

In the Fuzzy Neural Network, the aim oflearning algorithm adjust the linear output parameters, $p_{i i}, q_{i l}$ and $r_{i l}$ and the mean of the Gaussian function $m_{1, j}$ and $m_{2, k}$ and the variance of the Gaussian function $\sigma_{1, j}$ and $\sigma_{2, k}$.

This paper uses hybrid learning algorithm for learning Fuzzy Neural Network. The HybridLearning Algorithm is a combination ofleast square and backpropagation method.We use least square method for estimate linear output parameters, and backpropagation method for adjust gaussian nonlinear parameters. The equation of least square method as follows:

$y=\beta_{1} f_{1}(U)+\beta_{2} f_{2}(U)+\cdots \cdots+\beta_{n} f_{n}(U)$

Where $\mathrm{u}$ is input vector, $\beta_{i}$ are unknown parameters, $f_{i}$ are known function and $y$ is output. The equation (10) can be rewrite as follows

$$
A \beta=Y
$$

Where $A$ is $d \times n$ matrix. Thatd is the number of training data and $\mathrm{n}$ is number of unknown parameters in equation (10). Estimation of $\beta$ is $\hat{\beta}$ and is given by

$$
\begin{gathered}
\hat{\beta}= \\
\left(A^{T} A\right)^{-1} A^{T} Y
\end{gathered}
$$

By using equations (10-12), output linear parameters are obtained. Then by using back propagation method, as follows

$\alpha_{\text {new }}=\alpha_{\text {old }}+\Delta \alpha$

Where $\alpha$ is the parameter that to be optimized. In equation (13), $\Delta \alpha$ is given by

$\Delta \alpha=-\eta \frac{\partial E}{\partial \alpha}$

Where $E$ is difference between desired output $T_{m}$ and network output $\mathrm{T}$

$E(d)=\frac{1}{2}\left(T_{m}(d)-T(d)\right)^{2}=\frac{1}{2} e^{2}(d)$

By using beck propagation algorithm, The error expression for Layer 4 as follows

$\delta_{0}^{4}=-\frac{\partial E}{0_{0}^{4}}=\mathrm{e}$

The error expression for Layer 3as follows

$\delta_{\mathrm{jk}}^{3}=-\frac{\partial E}{\partial \mathrm{O}_{\mathrm{jk}}^{3}}=\frac{\partial E}{\mathrm{O}_{0}^{4}} \frac{\partial \mathrm{O}_{0}^{4}}{\partial \mathrm{O}_{\mathrm{jk}}^{3}}=\frac{1}{\mathrm{~b}} \delta_{0}^{4}$

The error expression for Layer 2 as follows

$\delta_{\mathrm{jk}}^{2}=-\frac{\partial E}{\partial 0_{j k}^{2}}=-\frac{\partial E}{\partial 0_{j \mathrm{k}}^{3}} \frac{\partial 0_{\mathrm{jk}}^{3}}{\partial 0_{\mathrm{ik}}^{2}}=\delta_{\mathrm{jk}}^{3} \mathrm{f}_{\mathrm{jk}}$

The error expression for Layer 1 as follows

$$
\left\{\begin{array}{l}
\delta_{1, \mathrm{j}}^{I I}=-\frac{\partial E}{\partial \mathrm{O}_{j k}^{I I}}=\sum_{\mathrm{k}} \delta_{\mathrm{jk}}^{I I I} \mathrm{w}_{\mathrm{jk}} \\
\delta_{2, \mathrm{k}}^{I I}=-\frac{\partial E}{\partial 0_{j \mathrm{k}}^{I I}}=\sum_{\mathrm{i}} \delta_{\mathrm{jk}}^{I I I} \mathrm{w}_{\mathrm{jk}}
\end{array}\right.
$$

The equations $13-20$ are the equation of update the antecedent parameters that they are the mean and the variance of gaussian function.

$$
\begin{array}{r}
\Delta m_{1, j}(k)=-\frac{\partial E}{\partial m_{1, j}}=-\frac{\partial E}{\partial \mu_{1, j}} \frac{\partial \mu_{1, j}}{\partial m_{1, j}} \\
=\delta_{1, j}^{I I} \frac{2\left(x_{1, j}-m_{1, j}\right)}{\left(\sigma_{1, j}\right)^{2}}
\end{array}
$$

$\Delta m_{2, k}(k)=-\frac{\partial E}{\partial m_{2, k}}=-\frac{\partial E}{\partial \mu_{2, k}} \frac{\partial \mu_{2, k}}{\partial m_{2, k}}$

$=\delta_{2, k}^{I I} \frac{2\left(x_{2, k}-m_{2, k}\right)}{\left(\sigma_{2, k}\right)^{2}}$

$\Delta \sigma_{1, j}(k)=-\frac{\partial E}{\partial \sigma_{1, j}}=-\frac{\partial E}{\partial \mu_{2, k}} \frac{\partial \mu_{2, k}}{\partial \sigma_{1, j}}=\delta_{2, k}^{I I} \frac{2\left(x_{1, j}-m_{1, j}\right)^{2}}{\left(\sigma_{1, j}\right)^{3}}$

$\Delta \sigma_{2, k}(k)=-\frac{\partial E}{\partial \sigma_{2, k}}=-\frac{\partial E}{\partial \mu_{2, k}} \frac{\partial \mu_{2, k}}{\partial \sigma_{2, k}}$

$=\delta_{2, k}^{I I} \frac{2\left(x_{2, k}-m_{2, k}\right)^{2}}{\left(\sigma_{2, k}\right)^{3}}$

$\left\{\begin{array}{l}m_{1, j}(k)=m_{1, j}(k-1)+\mu_{m} \Delta m_{1, j}(k) \\ m_{2, k}(k)=m_{2, k}(k-1)+\mu_{m} \Delta m_{2, k}(k)\end{array}\right.$
Jafar Tavoosi et al. Indian J.Sci.Technol. 


$$
\left\{\begin{array}{l}
\sigma_{1, j}(k)=\sigma_{1, j}(k-1)+\mu_{\sigma} \Delta \sigma_{1, j}(k) \\
\sigma_{2, k}(k)=\sigma_{2, k}(k-1)+\mu_{\sigma} \Delta \sigma_{2, k}(k)
\end{array}\right.
$$

\section{Simulation}

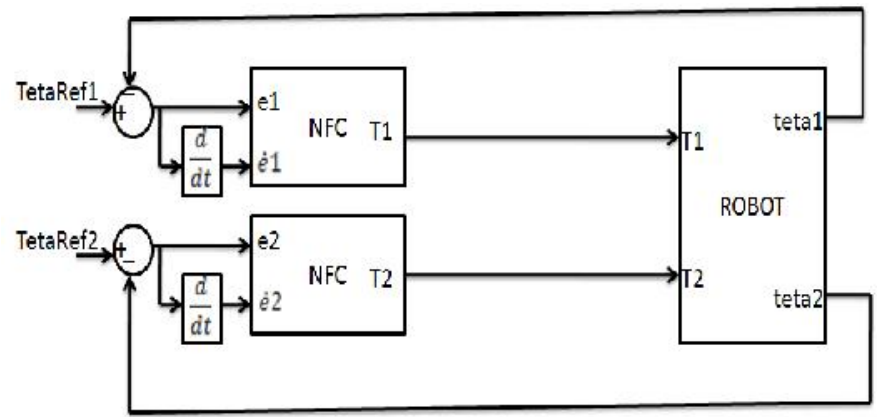

Fig 4. Simulink model of the NFC and robot

The block diagram of robot arm and neuro- fuzzy controller is shown in Fig.4. In this block, two trained fuzzy neural networks are used that one of them isutilized for control of $\theta_{1}$ and another is utilized for control of $\theta_{2}$. The initial value of $\theta_{1}$ and $\theta_{2}$ are [-30 -40](deg) and the final value of $\theta_{1}$ and $\theta_{2}$ are [30 40](deg), respectively.

Two Neuro - Fuzzy controllers are effective to track desired trajectory which are designed for two joints. These controllers have five membership functions and gaussian type membership functions are used in their fuzzification process. The rule bases of controllers are made of 25rules and these rules are determined by fuzzy neural network (FNN).

The desired position and the actual position for joints 1 and 2 are shown in Figs. 5 and 6, respectively.

The desired position and the actual position of the neuro-fuzzy and PID controller for joints 1 and 2 are given in Figs. 7 and 8 . These results show thatperformances of NFCs are better than PID controllers' performances over 2-DOF robot arm. Neuro- fuzzy controller has fast response and small errors fordifferent rise functions over trajectory control of robot arm.

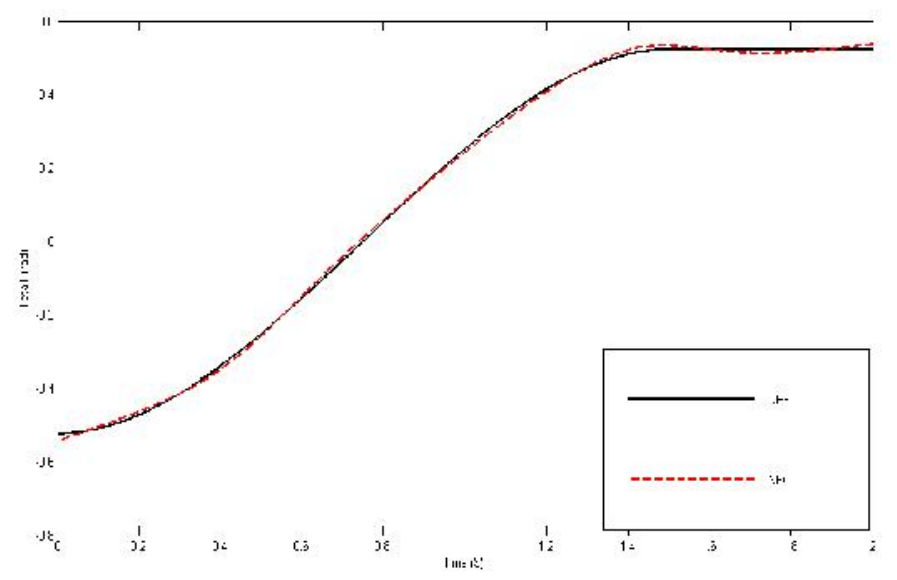

Fig. 5. The desired position and the actual position for joints 1

Mehmet ÖnderEfe (2008) uses a novel parameter adjustmentscheme to improve the robustness of fuzzy sliding-mode controlachieved by the use of an adaptive Research article COIndian Society for Education and Environment (iSee)
"Control of robot arm" http://www.indjst.org
Vol. 5 No.3 (Mar 2012)

ISSN: 0974- 6846

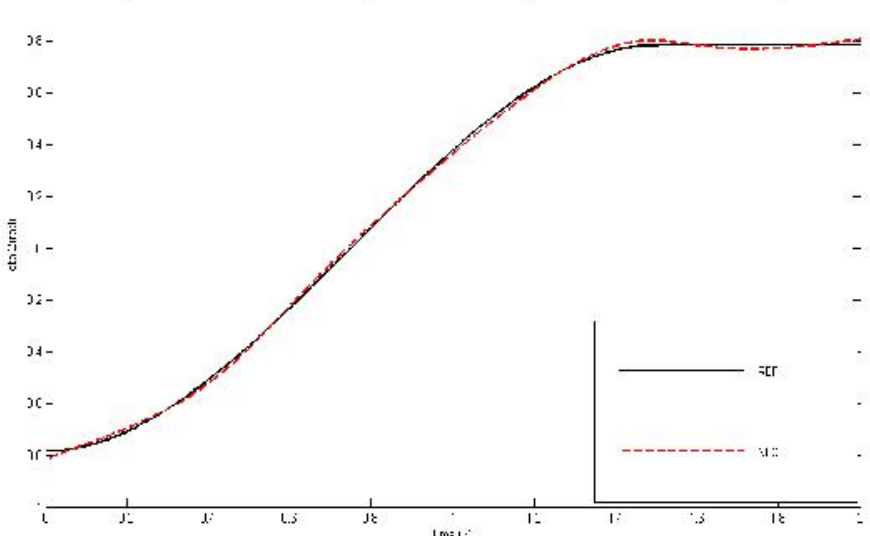

Fig. 6. The desired position and the actual position for joints 2

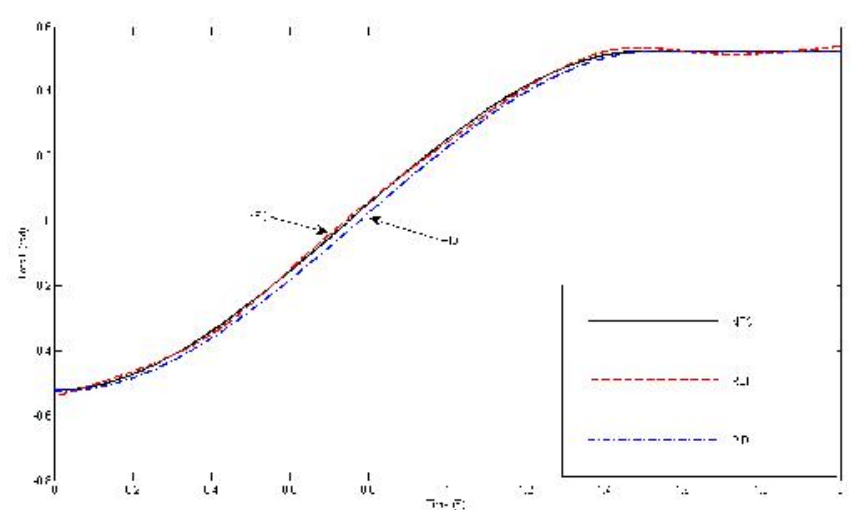

Fig. 7. The desired position and the actual position of the neuro-fuzzy and PID controller for joints $1\left(\theta_{1}\right)$

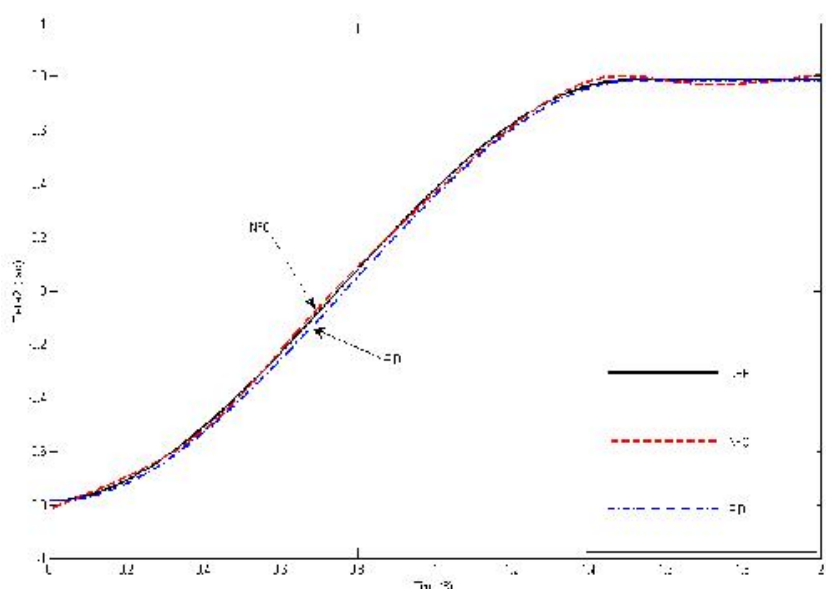

Fig.8. The desired position and the actual position of the neuro-fuzzy and PID controller for joints $2\left(\theta_{2}\right)$

neuro-fuzzy inference system(ANFIS) architecture. This paper has showed fuzzy sliding-mode control has better robustness and noise rejection capabilities than traditional integer-order operators, better tracking capability and better system response, better conditions for hitting infinite time and sliding-mode control based on 
fractional order adaptation. The crux of the approach is the use of sign equality in between the switching function defined for one subsystem and the error on the relevant control signal. Because the latter is not known due to the nature of the control problems, this paper demonstrates the conditions under which one can mention such equalities. For the fractional adaptation scheme, this paper provides an upper bound for the hitting time, and parallel to the claims, in the application example, it is shown that the presented form of the adaptation law provides, compared to its integer order counterpart. By demonstrating the usefulness of fractional-order operators in adaptation mechanisms, this paper addresses a wide range of applications from the field of adaptive control; more specifically, the field of adaptive sliding-mode control is focused in this paper.

To compare our paper to Mehmet ÖnderEfe (2008) can say that the proposed method in our paper is very easier than Mehmet ÖnderEfe's method. Our method is simple and communicative to understand, but Mehmet ÖnderEfe's method is very complex not simple because it uses of the combination of sliding mode control, fuzzy systems and neuro fuzzy networks. The performances of the proposed methods in both papers are same as or have a little difference.

\section{Conclusions}

This paper introduced two different NFC switch have designed for trajectory tracking control of a robot arm. NFCs have provided best results for control of robotic manipulators as compared to the conventional control strategies. From the simulation results, the joint-position tracking responses can be controlled to follow the reference trajectories accurately under a wide range of operating conditions and the occurrence of uncertainties. The NFC controller presented very interesting tracking features and was able to respond to different dynamic conditions. Inaddition, the fuzzy control computation is very inexpensive, and this regulator could be used for the control of machine tools and robotics manipulators without significantly increasing the cost of the drive. The proposed design confirms the fact that fuzzy control is relevant to the fast control of non-linear processes such as Robot manipulator control where quantitative methods are not always appropriate. Simulation results show that the Neuro-Fuzzy controller can achieve better accuracy and has less or no deviation from the trajectory compared to the PID controller. It is verified that the Neuro- Fuzzy controller has better control performance in robot trajectory control.

\section{References}

1. AnkaraliA, TinkirM and SahinY (2010) Neuro-Fuzzy Trajectory Control of AScaraRobot. The 2nd International Conference on Computer and Automation Engineering (ICCAE), pp: 298- 302.

2. AlavandarS, NigamMJ (2008) Inverse Kinematics Solution of 3DOF Planar Robot using ANFIS.Int.J.Comp.Commun.\& Cont. 3, 150-155.
3. Bingul $Z$ and Karahan O (2011) A Fuzzy Logic Controller tuned with PSO for 2 DOF robottrajectory control. J. Expert Sys. with Appl. 38, pp:1017-1031.

4. Deepak Batra, Sanjay Sharma and Rajeev Ratan (2009) Axis controlled movement of robot using brushless DC motor drive. Indian J. Sci. Technol. 4,pp: 36-39.

5. Jo-Anne Ting, Aaron D'Souza and Stefan Schaal (2011) Bayesian robot system identification with input and output noise.J. Neural Networks. 24, 99-108.

6. Jun Wu, Jinsong Wang and ZhengYou (2010) An overview of dynamic parameter identification of robots.J. Robotics \& Computer-Integrated Manufact. 26,414-419.

7. Kuo-Ho Su, Yih-Young Chen and Shun-Feng Su (2010)Design of neural-fuzzy-basedcontroller for two autonomously driven wheeled robot.J. Neurocomputing. 73, 2478-2488.

8. Mehmet ÖnderEfe (2008) Fractional fuzzy adaptive sliding-mode controlof a 2-DOF direct-drive robot arm. IEEE Trans. On Systems, Man, and CyberneticsPart B: Cybernetics, 38(6), 1561-1570.

9. NikpayN AliyariShM and Teshnehlab M (2010) Adaptive Intelligent Control of FlexibleLink Robot Arm, 8th Int. Symp. on Intelligent Systems and Informatics Sep. 10-11, Subotica, Serbia.pp: 653- 658.

10. Ouardal andWisamaKh (2010) Inverse and direct dynamic models of hybrid robots, Mechanism \& Machine Theory. 45(4), 627-640.
Jafar Tavoosi et al. Indian J.Sci.Technol. 